\title{
Ischemic preconditioning attenuates lipid peroxidation and apoptosis in the cecal ligation and puncture model of sepsis
}

\author{
ÇIMEN GÜLBEN OLGUNER ${ }^{1}$, UĞUR KOCA ${ }^{1}$, EMEL ALTEKIN $^{2}$, BEKIR UĞUR ERGÜR ${ }^{3}$, \\ SEDEN DURU ${ }^{1}$, PELIN GIRGIN ${ }^{4}$, AYDIN TAŞDÖĞEN ${ }^{1}$, KERIM GÜNDÜZ ${ }^{2}$, \\ SEDA GÜZELDAĞ ${ }^{1}$, MUHAMMED AKKUŞ ${ }^{5}$ and SERAP CILAKER MICILI ${ }^{3}$
}

\begin{abstract}
Departments of ${ }^{1}$ Anaesthesiology and Reanimation, ${ }^{2}$ Biochemistry and Clinical Biochemistry and ${ }^{3}$ Histology and Embryology, School of Medicine, Dokuz Eylül University, İzmir 35340; ${ }^{4}$ Department of Anaesthesiology and Reanimation, Şifa Hospital, İzmir 35100; ${ }^{5}$ Department of Anaesthesiology and Reanimation, Samandağ State Hospital, Hatay 31800, Turkey
\end{abstract}

Received January 8, 2013; Accepted March 19, 2013

DOI: $10.3892 /$ etm.2013.1034

\begin{abstract}
Sepsis and septic shock are are among the major causes of mortality in intensive care units. The lung and kidney are the organs most affected by sepsis. Evidence exists that lipid peroxidation and apoptosis may be responsible for the high mortality due to sepsis. Ischemic preconditioning (IP) is a method for the protection of tissues and organs against ischemia/reperfusion injury by reducing reactive oxygen species levels, lipid peroxidation and apoptosis. In the present study, the effects of IP were investigated in cecal ligation and puncture (CLP)-induced sepsis in rats. The three groups of animals used in the present controlled study were the sham-operated group (sham, $n=7$ ), which only underwent a laparotomy; the sepsis group (sepsis, $n=7$ ), which underwent cecal ligation and perforation; and the IP + sepsis group (IP+sepsis, $n=7)$, which underwent CLP immediately prior to the application of three cycles of IP to the hind limb. The study was terminated at $6 \mathrm{~h}$ after the induction of CLP. Blood, kidney and lung tissue samples were collected for the determination of serum creatinine, blood urea nitrogen (BUN), neutrophil gelatinase-associated lipocalin (NGAL) and lung tissue malondialdehyde (MDA) levels, as well as histological examination. The serum creatinine, plasma NGAL and lung tissue MDA levels in the sepsis group were significantly increased compared with those in the sham and the IP+sepsis groups $(\mathrm{P}<0.05)$. Alveolar macrophage counts, histological kidney and lung injury scores, kidney (caspase 3) and lung tissue immuonreactivity (M30) scores in the sepsis group were also significantly increased compared with those in the sham and $\mathrm{IP}+$ sepsis groups $(\mathrm{P}<0.05)$. The alveolar macrophage count in
\end{abstract}

Correspondence to: Professor Çimen Gülben Olguner, Department of Anaesthesiology and Reanimation, Dokuz Eylul University School of Medicine, Mithatpasa St, Inciralti, 35340, Izmir, Turkey E-mail: olgunercimen@gmail.com

Key words: ischemic preconditioning, lipid peroxidation, apoptosis, lung, kidney, sepsis the IP+sepsis group was increased compared with that in the sham group $(\mathrm{P}<0.05)$. In conclusion, IP inhibits lipid peroxidation and attenuates histological injury and apoptosis in the lung and kidney during sepsis.

\section{Introduction}

Severe sepsis is a major cause of mortality in the intensive care unit (ICU). Despite advances in supportive care and diagnostic tools, severe sepsis and septic shock remain associated with high mortality rates (25-54\%) (1-3). Although the induction of the inflammatory c-ascade in sepsis is mainly associated with infectious agents, it exhibits great similarity to ischemia/reperfusion (IR) injury in tissues. In particular, the free oxygen radicals that are generated during ischemia and the superoxide radicals that emerge during reperfusion cause endothelial injury, increased capillary permeability and tissue edema. The cytokines and adhesion molecules activated during IR initiate systemic inflammatory response syndrome (SIRS) which is also observed in sepsis (4-6).

Sepsis is one of the major extrapulmonary causes of acute lung injury (ALI) and acute respiratory distress syndrome (ARDS) (3). The pulmonary endothelium becomes lined with cytokine-containing proteins as a result of cytokine release, neutrophil activation and increased capillary permeability and reactive oxygen species (ROS) generation. ROS production at levels greater than the antioxidant capacity causes lipid peroxidation and consequently tissue and cell injury (7-9).

Another organ affected by sepsis is the kidney. Sepsis/septic shock causes acute kidney injury (AKI) via the modulation of renal inflammation by specific components of the sepsis-induced inflammatory cascade. Tubular cell apoptosis in the kidney may be significant in septic AKI $(10,11)$.

Ischemic preconditioning (IP) consists of brief ischemic periods that prepare the tissue or organs for a subsequent long period of ischemia. The excitement of the endogenous protective mechanisms against ischemia is the basis of IP. This phenomenon was described by Murry et al for the first time in 1986 (12). The protective molecular or cellular mechanisms and mediators of IP remain unclear. However, there is much evidence that IP modulates adenosine receptors, activates 
$\mathrm{K}$-ATP channels, decreases ROS production and reduces the expression of $\mathrm{TNF} \alpha$ and $\mathrm{NF} \kappa \mathrm{B}$ (13-15).

Remote IP is another method of conducting IP. The basic mechanism of remote IP is preparing one organ for ischemic insult by the application of IP to another site of the body (generally a lower limb) (16-18). Limb IP decreases the IR injuries in the lung, liver, myocardium and kidney and ameliorates the hemodynamic response to tourniquet application (19-27). These previous studies have shown that limb IP is a promising method for the prevention of IR-induced tissue injuries in certain clinical conditions. However, the effect of limb IP on sepsis-induced tissue injury has not yet been extensively studied. In only one study, the inflammatory response was shown to be modulated by intestinal IP in an endotoxemic shock model (28).

The cecal ligation and puncture (CLP) rodent model is widely used as it mimics human polymicrobial sepsis more closely than lipopolysaccharide (LPS) administration. According to evidence-based data, CLP creates an inflammatory response after inducing sepsis, by increasing TNF $\alpha$, IL-8 and ROS production, initiating apoptosis, decreasing mean arterial pressure and increasing lactate production (29-33)., However, limb IP appears to be a more feasible method for the treatment of the clinical conditions of patients with sepsis. Thus, we designed an experimental study using the CLP sepsis model in rats to evaluate the effects of limb IP on histological and biochemical parameters in the lung and kidney.

\section{Materials and methods}

Animals. A total of 21 adult male Wistar rats (body weight, 200-250 g) were used in the experiment. All animals were obtained from the Multidisciplinary Experimental Animal Laboratory at Dokuz Eylül University, School of Medicine, İzmir with the approval of the Animal Experiment Ethical Committee of Dokuz Eylül University, School of Medicine (İzmir, Turkey).

Animals were caged in groups of five with free access to food and water and were maintained on a 12-h light-dark cycle at a room temperature of $22 \pm 1^{\circ} \mathrm{C}$.

The rats were anesthetized with a mixture of $50 \mathrm{mg} / \mathrm{kg}$ ketamine (Ketalar ${ }^{\circledR}$, Pfizer Pharma GmbH, Berlin, Germany) and $10 \mathrm{mg} / \mathrm{kg}$ xylazine hydrochloride (Alfazyne ${ }^{\circledR}, 2 \%$; Alfasan International, Woerden, The Netherlands) that was administered intraperitoneally. The doses were repeated for the immobilization of the rats while maintaining spontaneous ventilation.

Experimental sepsis model by CLP. The rats were subjected to CLP as previously described $(34,35)$. Briefly, under aseptic conditions, a $3-\mathrm{cm}$ midline laparotomy was performed to allow the exposure of the cecum and adjoining intestine. The cecum was tightly ligated with a 2.0-silk suture at its base, below the ileocecal valve, and was perforated twice with an 18 -gauge needle. The cecum was then gently squeezed to extrude a small amount of feces from the puncture site. The cecum was then returned to the peritoneal cavity and the laparotomy was closed with 3.0-silk sutures. Sham-operated animals underwent the same surgical procedure although the cecum was neither ligated nor punctured. Saline $(3 \mathrm{ml} / 100 \mathrm{~g})$ was administered to all rats intraperitoneally at the end of the procedure. All animals were returned to their cages with free access to food and water.

$I P$. Left-lower-limb IP was performed by applying a rubber band tourniquet high around the left thigh for $10 \mathrm{~min}$ followed by reperfusion for $10 \mathrm{~min}$, as previously reported. The cessation of the blood flow during the ischemic period was confirmed with laser Doppler flowmetry. Three cycles of limb IP were performed to achieve effective pre-conditioning (19).

Experimental groups and protocol. The three groups of animals used in the present study were the sham-operated group (sham, $\mathrm{n}=7$ ), which underwent a laparotomy; the sepsis group (sepsis, $n=7$ ), which underwent CLP; and the IP-treated group (IP+sepsis, $n=7$ ), which underwent CLP immediately prior to the application of three cycles of IP.

The rats were kept at a constant environmental temperature of $37^{\circ} \mathrm{C}$ to maintain body heat following the procedures. At $6 \mathrm{~h}$ after the CLP, the rats were reanesthetized with the same dose of ketamine and xylazine hydrochloride, then their abdomens were opened and kidneys removed. Following a midline sternotomy, the rats were exsanguinated by needle aspiration of the right ventricle and their lungs were removed. Plasma samples and the left lungs were immediately transferred to a biochemistry laboratory and stored at $-80^{\circ} \mathrm{C}$ for the determination of serum creatinine, blood urea nitrogen (BUN), plasma neutrophil gelatinase-associated lipocalin (NGAL) and lung tissue malondialdehyde (MDA) levels. The right lungs and kidneys were fixed in $10 \%$ formalin solution for histomorphological determination and apoptosis (cytokeratin 18 with M30 immunostaining for lungs and caspase-3 immunostaining for kidneys).

Serum creatinine and BUN levels. Serum creatinine and BUN were measured with a is urine/CSF protein clinical chemistry kit (Abbott-Laboratories, Abbott Park, IL, USA) on an Architect 16000 analyzer (Abbott Laboratories, Chicago, IL, USA). BUN and serum creatinine results are expressed as $\mathrm{mg} / \mathrm{dl}$.

Detection of lung tissue lipid peroxidation. Tissue homogenates were prepared by the mechanical disruption of tissue samples using a TissueLyser (Qiagen, Hilden, Germany) for $5 \mathrm{~min}$ at $30 \mathrm{~Hz}$ in $0.1 \mathrm{M}$ phosphate buffer, $\mathrm{pH}$ 7.5. Tissue homogenates were then centrifuged at $10,000 \times \mathrm{g}, 4^{\circ} \mathrm{C}$ for $5 \mathrm{~min}$. The upper clear supernatants were transferred to a 2-ml Eppendorf tube. The protein levels of the tissue samples were measured with a quantitative kit using an Architect 16000 analyzer (Abbott Laboratories). The concentrations of MDA in the samples were determined using a high-pressure liquid chromatography method as described by Hong et al (36), using a $5 \mu \mathrm{M}$ C-18 reversed-phase column (250 x $4.6 \mathrm{~mm}$ I.D) and a mobile phase of $\mathrm{KH}_{2} \mathrm{PO}_{4}(0.01 \mathrm{M})$ and $30 \%$ methanol with a fluorescent detector. The MDA results are expressed in $\mu \mathrm{mol} / \mathrm{g}$ protein.

Serum neutrophil gelatinase-associated lipocalin. Serum NGAL levels were quantified using a commercially available NGAL ELISA kit (Boster Biological Technology, Wuhan, 
Table I. Serum creatinine, BUN, kidney injury and apoptosis (caspase 3) scores in all experimental groups.

\begin{tabular}{lcccc}
\hline Group & Serum creatinine $(\mathrm{mg} / \mathrm{dl})$ & BUN $(\mathrm{mg} / \mathrm{dl})$ & Kidney injury score $(0-4)$ & Caspase 3 score $(0-3)$ \\
\hline Sham & $0.50 \pm 0.22$ & $21.14 \pm 3.62$ & $0.28 \pm 0.48$ & $0.42 \pm 0.53$ \\
Sepsis & $0.58 \pm 0.05^{\mathrm{a}}$ & $29.28 \pm 3.99^{\mathrm{a}}$ & $1.71 \pm 0.48^{\mathrm{a}}$ & $1.57 \pm 0.53^{\mathrm{a}}$ \\
IP+sepsis & $0.52 \pm 0.03^{\mathrm{b}}$ & $24.00 \pm 3.82^{\mathrm{b}}$ & $0.57 \pm 0.78^{\mathrm{b}}$ & $0.85 \pm 0.37^{\mathrm{b}}$ \\
\hline
\end{tabular}

All data are the mean $\pm \mathrm{SD}(\mathrm{n}: 7)$. ${ }^{\mathrm{a}} \mathrm{P}<0.05$ compared with the sham group; ${ }^{\mathrm{b}} \mathrm{P}<0.05$ compared with the sepsis group. IP, ischemic preconditioning; BUN, blood urea nitrogen.

China) according to the manufacturer's instructions. The plasma NGAL results are expressed in $\mathrm{pg} / \mathrm{ml}$.

\section{Histomorphological procedures}

Light microscopic tissue preparation. The tissue samples were fixed in $10 \%$ formalin in phosphate buffer for $24 \mathrm{~h}$, then processed by routine histological methods and embedded in paraffin blocks. Sections of 4-5 $\mu \mathrm{m}$ thickness were obtained and stained with hematoxylin and eosin (H\&E), periodic acid-Schiff (PAS) and Masson's trichrome stains. The H\&E-stained sections were used to evaluate the general morphology; the PAS stain for the basal membrane and brush border of the tubules; and the Masson's trichrome stain for the collagen content of the parenchyma. All histomorphological and immunohistochemical assessments of the lungs and kidneys were evaluated by two histologists blinded to the groups.

Histomorphological assessment of lung tissue. Digital images were obtained from the H\&E- and Masson's trichrome-stained sections using a digital camera (DP71; Olympus, Tokyo, Japan) connected to a light microscope (Olympus BX51). Three non-overlapping lung sections and a minimum of 30 lung fields were examined per animal. A grading system was used to score the general alveolar and parenchymal morphological changes, including alveolar structure, inflammation, thickening of the alveolar septum, alveolar macrophages, neutrophils, increased capillary permeability, hemorrhage, edema and congestion. The grading system was scored according to these findings by the absence (score, 0 ), or presence (score, 1 for mild, 2 for moderate, 3 for marked and 4 for diffuse) of these changes in the alveolar tissue (37).

The number of alveolar macrophages in the alveolar septum and lumen was visualized digitally and counted in a given area (macrophage count $/ 0.016 \mathrm{~mm}^{2}$ ).

Histomorphological evaluation of kidney tissue. The sections were stained with H\&E and PAS stains. Three non-overlapping kidney sections and a minimum of 30 kidney fields were examined per animal. The structural changes in the kidney tissue sections were evaluated using light microscopy and scored for proximal tubule damage (tubular atrophy, tubular brush border loss, vacuolization, tubular dilation and cast formation), mononuclear cell infiltration, erythrocyte extravasation, interstitial structural changes, renal corpuscle morphology and necrotic and apoptotic cells. The tubulointerstitial damage in the obtained cross-sectional images was scored semiquantitatively. The scoring system for these findings was: 0 , none; $1,1-25 \% ; 2,26-50 \% ; 3,51-75 \%$; and 4 , $76-100 \%$ (30).

Determination of apoptosis by immunohistochemical study. To detect DNA fragmentation in epithelial cells in the lung tissue, M30 with cytokeratin-18 was used, while active caspase-3 immunohistochemistry was used to evaluate apoptosis in the kidney tissue in the paraffin sections. Following routine immunohistochemical procedures, the sections were incubated overnight at $4^{\circ} \mathrm{C}$ with rat-specific anti-M30 antibody (1:100, SC-32329; Santa Cruz Biotechnology, Inc., Santa Cruz, CA, USA) and active form anti-caspase-3 antibody (1:100, AB3623; Millipore, Temecula, CA, USA). The sections were then stained with DAB and counterstained with Mayer's hematoxylin. A grading system was used to score the quantity of anti-M30 and anti-caspase-3 positive staining in the sections (38). The score was defined as follows: 0, no immunoreactivity; 1 , little positive staining; 2 , moderate positive staining between grade 1 and grade $3 ; 3$, marked positive staining evenly distributed across the whole image.

Statistical analysis. The Statistical Package for the Social Sciences 15 (SPSS 15.0; SPSS, Inc., Chicago, IL, USA) software was used for statistical analysis. Kruskal-Wallis and post hoc Mann-Whitney U tests were used for the comparison of biochemical results. The Chi-square test was used for comparison of the histopathological results. $\mathrm{P}<0.05$ was considered to indicate a statistically significant difference. The results are expressed as the mean $\pm \mathrm{SD}$.

\section{Results}

\section{Evaluation of the kidney}

Biochemical results. The serum creatinine and BUN levels were significantly higher in the sepsis group compared with those in the IP+sepsis and sham groups $(\mathrm{P}<0.05)$. No significant differences were observed between the IP+sepsis and the sham groups (Table I).

NGAL levels in the sepsis group $(52.05 \pm 11.67 \mathrm{pg} / \mathrm{ml})$ were significantly higher than those in the sham $(30.66 \pm 5.06 \mathrm{pg} / \mathrm{ml})$ and $\mathrm{IP}+$ sepsis $(38.17 \pm 9.15 \mathrm{pg} / \mathrm{ml})$ groups $(\mathrm{P}<0.05$; Fig. 1$)$. No significant differences were observed between the sham and $\mathrm{IP}+$ sepsis groups.

Histomorphological and immunohistochemical results. The histological kidney injury and kidney tissue immuonreactivity (caspase 3 ) scores in the sepsis group were significantly increased compared with those in the sham and IP+sepsis groups $(\mathrm{P}<0.05)$. The kidney injury and the kidney tissue 
Table II. Histological lung injury and apoptosis (M30) scores in all experimental groups.

\begin{tabular}{lcc}
\hline Group & Lung injury score (0-4) & M30 score (0-3) \\
\hline Sham & $0.14 \pm 0.37$ & $0.42 \pm 0.53$ \\
Sepsis & $2.00 \pm 0.57^{\mathrm{ab}}$ & $2.00 \pm 0.57^{\mathrm{ab}}$ \\
IP+sepsis & $0.71 \pm 0.71^{\mathrm{a}}$ & $0.85 \pm 0.37$ \\
\hline
\end{tabular}

All data are the mean $\pm \mathrm{SD}(\mathrm{n}=7) .{ }^{\mathrm{a}} \mathrm{P}<0.05$ compared with the sham group; ${ }^{b} \mathrm{P}<0.05$ compared with the $\mathrm{IP}+$ sepsis group. IP, ischemic preconditioning.

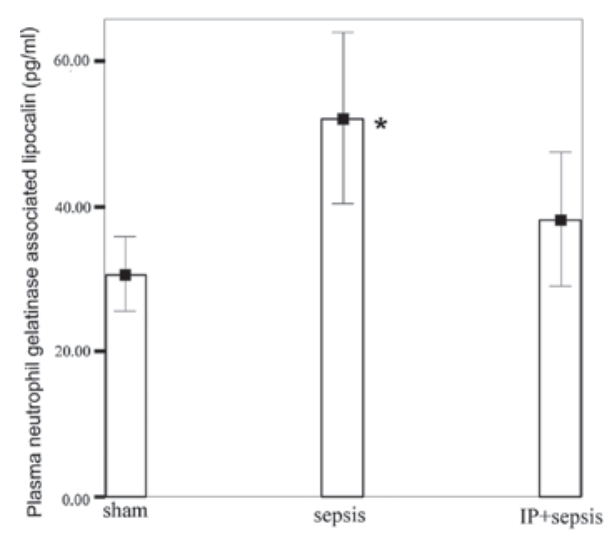

Figure 1. Plasma neutrophil gelatinase-associated lipocalin levels in all experimental groups. ${ }^{*} \mathrm{P}<0.05$ compared with the sham and IP+sepsis groups. IP, ischemic preconditioning.

immunoreactivity (caspase 3) scores in the IP+sepsis group were not significantly different from those in the sham group $(\mathrm{P}>0.05$; Table I).

Kidney photomicrographs. In the stained kidney tissue sections, the sham group showed a normal kidney structure (Fig. 2Aa,Ba and $\mathrm{Ca}$ ). In the sepsis group, mononuclear cell infiltration around the glomeruli and capillaries, vasodilation, scattered tubular degeneration and cast formation in the tubules were observed (Fig. 2Ab). In the PAS-stained sections, loss of the brush border and irregularity in the basal membrane were noted in the proximal tubular cells of the sepsis group (Fig. 2Bb). In the IP+sepsis group, the histomorphological changes were less evident compared with those in the sepsis group (Fig. 2Ac and $\mathrm{Bc}$ ). In the active caspase-3 immunohistochemical staining, the number of caspase-3 immunopositive cells was higher in the tissue sections of the sepsis group than in those of the sham and IP+sepsis groups (Fig. 2Cb and Cc).

\section{Evaluation of the lung}

Determination of lipid peroxidation. The lung tissue MDA levels were increased significantly in the sepsis group $(10.02 \pm 1.66 \mu \mathrm{mol} / \mathrm{g}$ protein) compared with those in the sham $(7.90 \pm 1.39 \mu \mathrm{mol} / \mathrm{g}$ protein) and IP+sepsis $(8.14 \pm 1.05 \mu \mathrm{mol} / \mathrm{g}$ protein) groups $(\mathrm{P}<0.05)$. No significant differences were detected between the IP+sepsis and sham groups (Fig. 3).
Histomorphological and immunohistochemical results. The histological lung injury and lung tissue immunoreactivity (M30) scores in the sepsis group were significantly increased compared with those in the sham and IP+sepsis groups $(\mathrm{P}<0.05)$. There were no differences in the lung tissue immunoreactivity (M30) scores between the IP+sepsis and sham groups. The lung injury score in the IP+sepsis group was elevated compared with that in the sham group (Table II). The alveolar macrophage count in the sepsis group $(19.57 \pm 1.81)$ was significantly increased compared with those in the sham $(8.00 \pm 0.81)$ and $\mathrm{IP}+$ sepsis $(14.57 \pm 1.71)$ groups $(\mathrm{P}<0.05)$ and was higher in the IP+sepsis group than in the sham group ( $\mathrm{P}<0.05$; Fig. 4).

Lung photomicrographs. The staining of the lung tissue sections showed that the sham group had normal lung histology (Fig. 5Aa, Bb and Cc). By contrast, there was a significant increase in the lung injury score following sepsis, with the development of alveolar wall thickening and inflammatory infiltration (Fig. 5Ab and Bb). When the sections from the sepsis group were observed, inflammation and thickening of the alveolar septum, particularly increases in alveolar macrophage and mononuclear-cell infiltration which ensured that the alveolar septum increased capillary permeability, hemorrhage, edema and congestion, were noted (Fig. 5Bb). However, in the IP+sepsis group, the structural lung injury was less severe compared with that in the sepsis group (Fig. 5Ac and $\mathrm{Bc}$ ). In the Masson's trichrome-stained sections, increased collagen content in the parenchyma of the lung tissue sections of the sepsis group was observed (Fig. 5Bb). Furthermore, in the M30 immunohistochemistry scoring, the number of M30 immunopositive epithelial cells was increased in the sepsis group compared with the numbers in the sham and IP+sepsis groups (Fig. $5 \mathrm{Cb}$ and $\mathrm{Cc}$ ).

\section{Discussion}

In the present study, it was shown histologically and biochemically that IP applied to the unilateral hind limb attenuated septic lung and kidney injury. To the best of the author's knowledge, the present study is the first to evaluate remote IP with regard to lung and kidney injury in a CLP sepsis model. CLP was selected for the generation of sepsis as it is a widely used experimental sepsis model that mimics human polymicrobial sepsis $(34,39)$.

Experimental and clinical studies have shown that IP increases resistance against ischemic tissue injury to the organs, including the heart, lung, kidney, intestine, liver and muscle $(20,25,28,40,41)$. SIRS may be induced by infection as well as IR. The mechanisms by which infections and IR trigger SIRS are almost the same. Therefore, it has been hypothesized that either ischemic or pharmacologically-simulated preconditioning may lead to new possibilities in the treatment of critically ill patients with sepsis and multiple organ dysfunction syndrome (MODS) $(4,24)$.

The main ischemic conditioning methods are preconditioning (prior to major ischemia), perconditioning (during major ischemia) and postconditioning (during reperfusion) which are named according to the application time (42). In the present study, IP was initiated immediately following the CLP procedure and completed within $1 \mathrm{~h}$. It has been shown 

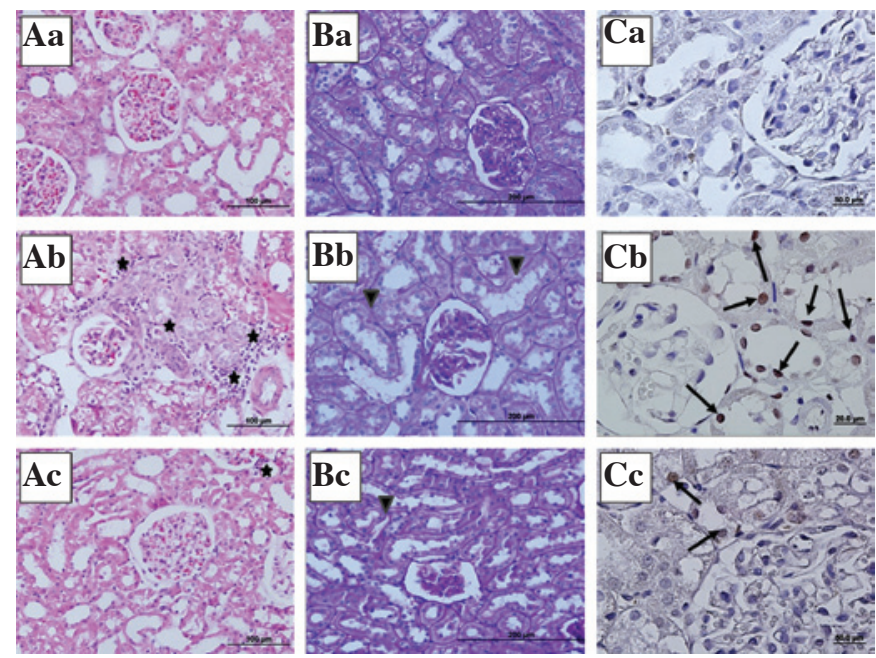

Figure 2. Representative micrographs of kidney tissue sections stained histochemically and immunohistochemically (H\&E, PAS and active caspase-3 staining). (A) H\&E (magnification, x20), (B) PAS (magnification, x10) and (C) active caspase-3 immunohistochemically stained sections (magnification, x40) from the (a) sham, (b) sepsis and (c) IP+sepsis groups. * mononuclear cell infiltration; $\mathbf{v}$, irregular basal membrane and brush border loss in proximal tubular epithelium; $\rightarrow$, active caspase- 3 immunpositive cells; H\&E, hematoxylin and eosin; PAS, periodic acid-Schiff; IP, ischemic preconditioning.

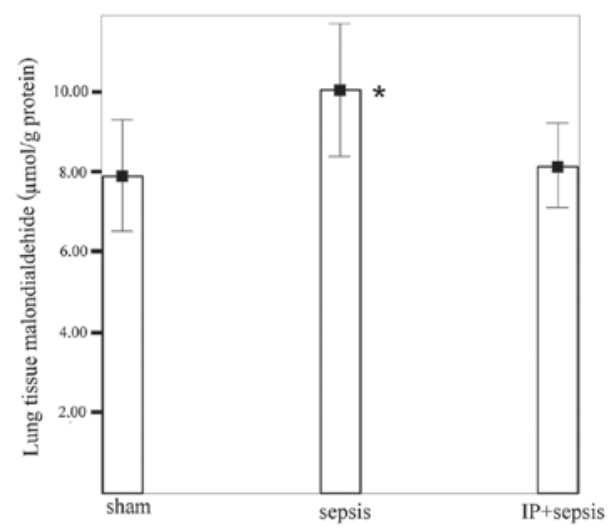

Figure 3. Lung tissue malondialdehyde levels in all experimental groups ${ }^{*} \mathrm{P}<0.05$ compared with the sham and IP+sepsis groups. IP, ischemic preconditioning.

that sepsis occurs $5 \mathrm{~h}$ after CLP $(35,43)$. Therefore the present method of ischemic conditioning was described as ischemic 'preconditioning'. The effectiveness of IP is associated with the number of ischemic cycles and length of the ischemic period $(17,44)$. In the present study, three $10 \mathrm{~min}$ IP cycles were used, for which effectiveness against IR had already been demonstrated (19), and the blood and tissue samples were collected $6 \mathrm{~h}$ after the CLP procedure.

The kidney is the one of the main organs affected during sepsis. Decreased renal perfusion and numbers of cortical capillaries, peaking of renal tubular apoptosis and increased leukocyte infiltration and ROS production have been observed in experimental models 4-6 h after the induction of sepsis $(29,32,45,46)$.

The protective effects of limb IP are transferred by means of heat shock proteins, nitric oxide, adenosine, bradykinin and



Figure 4. Alveolar macrophage count in all experimental groups. ${ }^{*} \mathrm{P}<0.05$ compared with the sham and IP+sepsis groups. ${ }^{\text {\& }} \mathrm{P}<0.05$ compared with the sham group. IP, ischemic preconditioning.
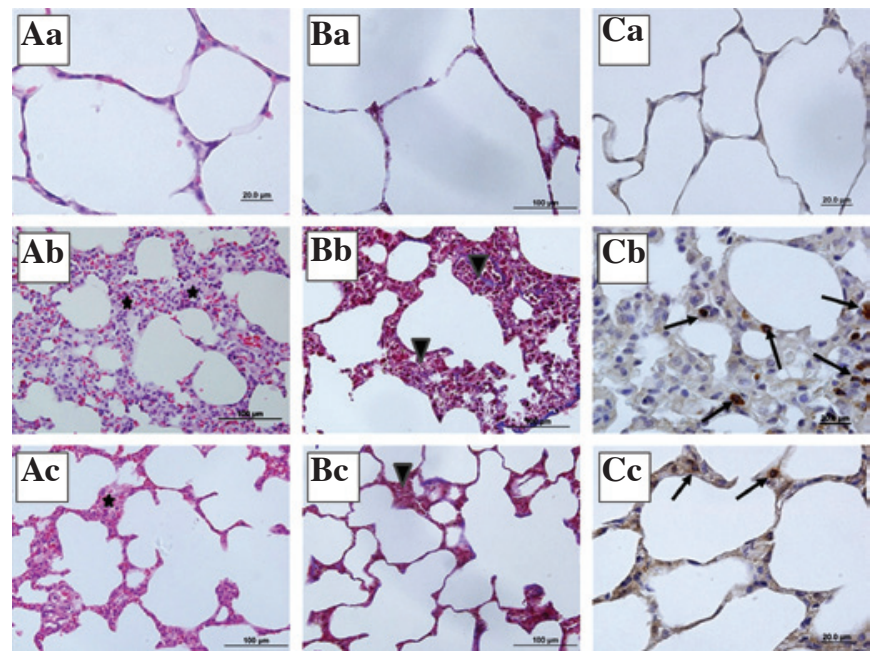

Figure 5. Representative micrographs of lung tissue sections stained histochemically and immunohistochemically (H\&E, Masson's trichrome and M30 staining). (A) H\&E (magnification, x40), (B) Masson's trichrome (magnification, x20), (C) M30 immunohistochemically stained sections (magnification, $\mathrm{x} 20$ ) from the (a) sham, (b) sepsis and (c) IP+sepsis groups. *, mononuclear cell infiltration; $\mathbf{v}$, collagen content of the parenchyma; $\rightarrow$, marked M30 immunopositive cells; H\&E, hematoxylin and eosin; IP, ischemic preconditioning.

neurogenic signals to the target organs $(13,47)$. The results of these interactions are reductions in ROS levels, reductions in ATP depletion and increases in ATP production which consequently increase the oxidative stress resistance of the cell $(13,15)$. The effect of limb IP on IR injury to the kidney has been shown to reduce serum creatinine and BUN levels (48). Er et al (49) and Zimmerman et al (25) demonstrated similar results in clinical studies. In the present study, it was observed that the BUN and serum creatinine levels were significantly higher in the sepsis group than in the IP+sepsis group, while no significant differences were observed between the IP+sepsis and sham groups. These findings indicate that IP protects renal function from sepsis-related kidney insult. This effect of limb IP may be associated with the reduction of ROS production or an increase in microvascular circulation.

NGAL is a highly sensitive, specific and predictive early biomarker for AKI $(50,51)$. Bagshaw et al (52) observed that 
patients with acute kidney sepsis have higher plasma and urine NGAL levels compared with patients without acute septic kidney injury. Furthermore, serum NGAL levels have been observed to be elevated in SIRS, sepsis and septic shock $(53,54)$. NGAL is present in the kidney, liver, spleen, lung and trachea, indicating that serum NGAL may not be a reliable marker of septic AKI (55). By contrast, Chen et al (14) showed that a major cause of elevated serum NGAL in the kidney is ischemic renal injury and the application of IP to the kidney decreases NGAL levels. Therefore, it may be concluded that the elevation of the plasma NGAL level in the present sepsis group may be associated not only with the kidney, but also other NGAL-containing tissues such as the lung, spleen and liver. However, the increase in the plasma NGAL level of the sepsis group reflected the sepsis-induced NGAL release from tissues which occurred in the present study. The present study also showed that the sepsis-associated NGAL release from tissues was efficiently inhibited by limb IP.

In sepsis, neutrophil accumulation in the lung is followed by cytokine and ROS production. ROS production exceeding the antioxidant capacity causes lipid peroxidation and consequent tissue injury and cell death $(9,56)$. MDA is the end-product of fatty acid peroxidation and is generated within inflammatory cells. The MDA content of the lungs increases significantly following CLP-induced sepsis $(43,57,58)$. Numerous studies have shown that IP diminishes neutrophil accumulation, lipid peroxidation and MDA levels in the lung (19,23,59-61). In the present study, the lung MDA levels were significantly higher in the sepsis group than in the IP+sepsis and sham groups. We conclude that remote IP has an antioxidant effect in CLP-induced sepsis by decreasing lipid peroxidation levels in the lung

Previous studies have investigated lung and kidney injuries using H\&E staining and light microscopy. Notably high histological lung and kidney injury scores have been observed in septic rats (62-65). IP attenuates lung and kidney histological injuries in ischemic conditions and Lee et al (66) histologically showed that hepatic IP is able to attenuate renal ischemic injury. It has been demonstrated that IP acts via A1 adenosine receptor activation $(15,41)$. The activation of the A1 adenosine receptor diminishes renal inflammation, apoptosis and necrosis in renal ischemia $(16,67)$.

We have previously demonstrated that unilateral hind limb IR causes lung injury and remote IP decreases the lung injury score (19). Similarly, lung injury has been mitigated using limb injury in the hemorrhagic shock model (5). In the present study it was observed that the lung and kidney histological injury scores were significantly lower in the IP+sepsis group than in the sepsis group. Therefore, we propose that IP reduces sepsis-induced lung and kidney injury not only biochemically, but also histologically.

The mononuclear phagocyte system is involved in phagocytosis and numerous complex immunological and inflammatory processes. Sepsis is associated with the increased production of cellular proinflammatory and inflammatory mediators by monocytes/macrophages. TNF $\alpha$ is the main mediator and has an important role in acute kidney and lung injury in sepsis $(68,69)$. Remote IP decreases the levels of TNF $\alpha$ and other inflammatory cytokines, including IL-6 and IL-8, in lung tissue and plasma, $(61,70)$. However, ROS activate the transcrip- tion factor $\mathrm{NF \kappa B}$ which stimulates the excessive production of inflammatory cytokines. Takeshita et al (71) showed that direct IP reduced NFKB activity and cytokine mRNA levels.

In the present study, a significantly higher alveolar macrophage count was observed in the sepsis group than in the sham and IP+sepsis groups. We were unable to measure the cytokine levels, which was a limitation of the present study. However, the CLP-induced alveolar macrophage count in the lung was decreased by limb IP. This effect may be explained by the reduction of TNF $\alpha$ levels and the inhibition of NFKB activity.

Apoptosis is a type of programmed cell death in which DNA disintegration and cell death occur as a result of the activation of death-inducing receptors or intracellular specific serine proteases (caspases) (72,73).

The gold standard for the diagnosis of apoptosis is morphological/ultrastructural evaluation. The determination of apoptosis using H\&E staining and light microscopy is sensitive and specific, while also being the least expensive approach $(74,75)$. However, to evaluate acute injury such as in CLP-induced sepsis, more early markers that indicate apoptosis are required. Therefore, caspase 3 and M30 (caspase cleaved cytokeratin 18 neo-epitope) staining were used in the present study to show kidney and lung epithelial apoptosis, respectively. The sensitivity of these two methods has been demonstrated clinically and experimentally, including in CLP-induced sepsis (11,57,76-78).

Apoptosis occurs through inflammatory cytokines and $\mathrm{TNF} \alpha$, apoptosis-associated proteins and ROS-mediated pathways during sepsis $(69,79-82)$.

IP reduces apoptosis by regulating the genes which encode these proteins and releasing the metabolites involved in the apoptotic process (83). The protective effect of IP on IR injury-induced apoptosis has been demonstrated in the lung and kidney $(80,83)$.

In the present study, significant increases were observed in the M30-positive cell count of the sepsis group compared with the sham group, indicating the presence of epithelial cell-specific apoptosis in sepsis. Also, no significant changes were noted in the number of pulmonary M30-positive cells in the IP+sepsis group compared with the sham group, suggesting that limb IP inhibits apoptosis in CLP-induced sepsis. Additionally, it was also observed that number of caspase-3-stained renal tubular cells was significantly lower in the IP+sepsis group than in the sepsis group and not significantly different between the sham and IP+sepsis groups. Jacobs et al (10) suggested that intrarenal inflammation is decreased by caspase inhibition through unclear mechanisms. It is possible that IP inhibits caspase. Together, these results suggest that IP attenuates apoptosis in the kidney and the lung during sepsis.

In conclusion, the present study presents evidence for the effectiveness of IP in the management of sepsis. Remote IP was observed to reduce lung lipid peroxidation, kidney and lung injuries and apoptosis in a CLP-induced sepsis model. Further investigations are required to fully elucidate the underlying mechanisms of IP in sepsis.

\section{References}

1. Angus DC, Linde-Zwirble WT, Lidicker J, Clermont G, Carcillo J and Pinsky MR: Epidemiology of severe sepsis in the United States: analysis of incidence, outcome, and associated costs of care. Crit Care Med 29: 1303-1310, 2001. 
2. Martin GS, Mannino DM, Eaton S and Moss M: The epidemiology of sepsis in the United States from 1979 through 2000. N Engl J Med 348: 1546-1554, 2003.

3. Vincent JL, Sakr Y, Sprung CL, et al; Sepsis Occurrence in Acutely Ill Patients Investigators: Sepsis in European intensive care units: results of the SOAP study. Crit Care Med 34: 344-353, 2006.

4. Rock $\mathrm{P}$ and Yao Z: Ischemia reperfusion injury, preconditioning and critical illness. Curr Opin Anaesthesiol 15: 139-146, 2002.

5. Jan WC, Chen CH, Tsai PS and Huang CJ: Limb ischemic preconditioning mitigates lung injury induced by haemorrhagic shock/resuscitation in rats. Resuscitation 82: 760-766, 2011.

6. Jan F, Burne M, O'Donnell M and Rabb H: Pathophysiologic role of selectins and their ligands in ischemia reperfusion injury. Front Biosci 5: E103-E109, 2000.

7. Hotchkiss RS and Karl IE: The pathophysiology and treatment of sepsis. N Engl J Med 348: 138-150, 2003.

8. Cohen J: The immunopathogenesis of sepsis. Nature 420: 885-891, 2002

9. Lange M, Szabo C, Traber DL, et al: Time profile of oxidative stress and neutrophil activation in ovine acute lung injury and sepsis. Shock 37: 468-472, 2012.

10. Jacobs R, Honore PM, Joannes-Boyau O, et al: Septic acute kidney injury: the culprit is inflammatory apoptosis rather than ischemic necrosis. Blood Purif 32: 262-265, 2011.

11. Lerolle N, Nochy D, Guérot E, et al: Histopathology of septic shock induced acute kidney injury: apoptosis and leukocytic infiltration. Intensive Care Med 36: 471-478, 2010.

12. Murry CE, Jennings RB and Reimer KA: Preconditioning with ischemia: a delay of lethal cell injury in ischemic myocardium. Circulation 74: 1124-1136, 1986.

13. Szijártó A, Czigány Z, Turóczi Z and Harsányi L: Remote ischemic perconditioning - a simple, low-risk method to decrease ischemic reperfusion injury: models, protocols and mechanistic background. A review. J Surg Res 178: 797-806, 2012

14. Chen X, Liu X, Wan X, Wu Y, Chen Y and Cao C: Ischemic preconditioning attenuates renal ischemia-reperfusion injury by inhibiting activation of IKKbeta and inflammatory response. Am J Nephrol 30: 287-294, 2009.

15. Riksen NP, Smits P and Rongen GA: Ischaemic preconditioning: from molecular characterisation to clinical application - part I. Neth J Med 62: 353-363, 2004.

16. Souza Filho MV, Loiola RT, Rocha EL, et al: Hind limb ischemic preconditioning induces an anti-inflammatory response by remote organs in rats. Braz J Med Biol Res 42: 921-929, 2009.

17. Kharbanda RK, Mortensen UM, White PA, et al: Transient limb ischemia induces remote ischemic preconditioning in vivo. Circulation 106: 2881-2883, 2002.

18. Takaoka A, Nakae I, Mitsunami K, et al: Renal ischemia/reperfusion remotely improves myocardial energy metabolism during myocardial ischemia via adenosine receptors in rabbits: effects of 'remote preconditioning'. J Am Coll Cardiol 33: 556-564, 1999.

19. Olguner C, Koca U, Kar A, et al: Ischemic preconditioning attenuates the lipid peroxidation and remote lung injury in the rat model of unilateral lower limb ischemia reperfusion. Acta Anaesthesiol Scand 50: 150-155, 2006

20. Harkin DW, Barros D'Sa AA, McCallion K, Hoper M and Campbell FC: Ischemic preconditioning before lower limb ischemia - reperfusion protects against acute lung injury. J Vasc Surg 35: 1264-1273, 2002.

21. Sahin E, Olguner C, Bodur HA, Koca U, Tuncel P, Örmen M, et al: Comparison of the effects of the remote and direct ischemic preconditioning in the liver ischemia-reperfusion injury. Türkiye Klinikleri. Tip Bilimleri Dergisi 29: 381-387, 2009 (In Turkish).

22. Lai IR, Chang KJ, Chen CF and Tsai HW: Transient limb ischemia induces remote preconditioning in liver among rats: the protective role of heme oxygenase-1. Transplantation 81: 1311-1317, 2006.

23. Granfeldt A, Jiang R, Wang NP, et al: Neutrophil inhibition contributes to cardioprotection by postconditioning. Acta Anaesthesiol Scand 56: 48-56, 2012

24. Xiong J, Liao X, Xue FS, Yuan YJ, Wang Q and Liu JH: Remote ischemia conditioning-an endogenous cardioprotective strategy from outside the heart. Chin Med J (Engl) 124: 2209-2215, 2011

25. Zimmerman RF, Ezeanuna PU, Kane JC, et al: Ischemic preconditioning at a remote site prevents acute kidney injury in patients following cardiac surgery. Kidney Int 80: 861-867, 2011

26. Van M, Olguner C, Koca U, et al: Ischaemic preconditioning attenuates haemodynamic response and lipid peroxidation in lower-extremity surgery with unilateral pneumatic tourniquet application: a clinical pilot study. Adv Ther 25: 355-366, 2008.
27. Lin LN, Wang LR, Wang WT, et al: Ischemic preconditioning attenuates pulmonary dysfunction after unilateral thigh tourniquet-induced ischemia-reperfusion. Anesth Analg 111: 539-543, 2010

28. Tamion F, Richard V, Renet S and Thuillez C: Intestinal preconditioning prevents inflammatory response by modulating heme oxygenase-1 expression in endotoxic shock model. Am J Physiol Gastrointest Liver Physiol 293: G1308-G1314, 2007.

29. Seely KA, Holthoff JH, Burns ST, et al: Hemodynamic changes in the kidney in a pediatric rat model of sepsis-induced acute kidney injury. Am J Physiol Renal Physiol 301: F209-F217, 2011.

30. Yasuda H, Yuen PS, Hu X, Zhou H and Star RA: Simvastatin improves sepsis-induced mortality and acute kidney injury via renal vascular effects. Kidney Int 69: 1535-1542, 2006.

31. Wu L, Gokden N and Mayeux PR: Evidence for the role of reactive nitrogen species in polymicrobial sepsis-induced renal peritubular capillary dysfunction and tubular injury. J Am Soc Nephrol 18: 1807-1815, 2007.

32. Messaris E, Memos N, Chatzigianni E, et al: Apoptotic death of renal tubular cells in experimental sepsis. Surg Infect (Larchmt) 9: 377-388, 2008.

33. Collin S, Sennoun N, Dron AG, et al: Vascular ATP-sensitive potassium channels are over-expressed and partially regulated by nitric oxide in experimental septic shock. Intensive Care Med 37: 861-869, 2011.

34. Wichterman KA, Baue AE and Chaudry IH: Sepsis and septic shock - a review of laboratory models and a proposal. J Surg Res 29: 189-201, 1980.

35. Otero-Antón E, González-Quintela A, López-Soto A, López-Ben S, Llovo J and Pérez LF: Cecal ligation and puncture as a model of sepsis in the rat: influence of the puncture size on mortality, bacteremia, endotoxemia and tumor necrosis factor alpha levels. Eur Surg Res 33: 77-79, 2001.

36. Hong YL, Yeh SL, Chang CY and Hu ML: Total plasma malondialdehyde levels in 16 Taiwanese college students determined by various thiobarbituric acid tests and an improved high-performance liquid chromatography-based method. Clin Biochem 33: 619-625, 2000.

37. Albaiceta GM, Gutiérrez-Fernández A, Parra D, et al: Lack of matrix metalloproteinase-9 worsens ventilator-induced lung injury. Am J Physiol Lung Cell Mol Physiol 294: L535-L543, 2008.

38. Tüzün F, Gencpinar P, Ozbal S, et al: Neuroprotective effect of neotrofin in a neonatal rat model of periventricular leukomalacia. Neurosci Lett 520: 6-10, 2012.

39. Holly MK, Dear JW, Hu X, et al: Biomarker and drug-target discovery using proteomics in a new rat model of sepsis-induced acute renal failure. Kidney Int 70: 496-506, 2006.

40. Peralta C, Closa D, Xaus C, Gelpí E, Roselló-Catafau J and Hotter G: Hepatic preconditioning in rats is defined by a balance of adenosine and xanthine. Hepatology 28: 768-773, 1998.

41. Riksen NP, Smits P and Rongen GA: Ischaemic preconditioning: from molecular characterisation to clinical application - part II. Neth J Med 62: 409-423, 2004

42. Vinten-Johansen J and Shi W: Perconditioning and postconditioning: current knowledge, knowledge gaps, barriers to adoption, and future directions. J Cardiovasc Pharmacol Ther 16: 260-266, 2011.

43. Hsu DZ and Liu MY: Effects of sesame oil on oxidative stress after the onset of sepsis in rats. Shock 22: 582-585, 2004.

44. Saita Y, Yokoyama K, Nakamura K and Itoman M: Protective effect of ischaemic preconditioning against ischaemia-induced reperfusion injury of skeletal muscle: how many preconditioning cycles are appropriate? Br J Plast Surg 55: 241-245, 2002.

45. Legrand M, Bezemer R, Kandil A, Demirci C, Payen D and Ince $\mathrm{C}$ : The role of renal hypoperfusion in development of renal microcirculatory dysfunction in endotoxemic rats. Intensive Care Med 37: 1534-1542, 2011

46. Wang Z, Holthoff JH, Seely KA, et al: Development of oxidative stress in the peritubular capillary microenvironment mediates sepsis-induced renal microcirculatory failure and acute kidney injury. Am J Pathol 180: 505-516, 2012.

47. Shihab FS: Preconditioning: from experimental findings to novel therapies in acute kidney injury. Minerva Urol Nefrol 61: 143-157, 2009

48. Kadkhodaee M, Seifi B, Najafi A and Sedaghat Z: First report of the protective effects of remote per- and postconditioning on ischemia/reperfusion-induced renal injury. Transplantation 92: e55, 2011 
49. Er F, Nia AM, Dopp H, et al: Ischemic preconditioning for prevention of contrast medium-induced nephropathy: randomized pilot RenPro Trial (Renal Protection Trial). Circulation 126: 296-303, 2012

50. Devarajan P: Emerging biomarkers of acute kidney injury. Contrib Nephrol 156: 203-212, 2007.

51. Dent CL, Ma Q, Dastrala S, et al: Plasma neutrophil gelatinase-associated lipocalin predicts acute kidney injury, morbidity and mortality after pediatric cardiac surgery: a prospective uncontrolled cohort study. Crit Care 11: R127, 2007.

52. Bagshaw SM, Bennett M, Haase M, et al: Plasma and urine neutrophil gelatinase-associated lipocalin in septic versus non-septic acute kidney injury in critical illness. Intensive Care Med 36: 452-461, 2010.

53. Han M, Li Y, Liu M and Cong B: Renal neutrophil gelatinase associated lipocalin expression in lipopolysaccharide-induced acute kidney injury in the rat. BMC Nephrol 13: 25, 2012.

54. Mårtensson J, Bell M, Oldner A, Xu S, Venge P and Martling CR: Neutrophil gelatinase-associated lipocalin in adult septic patients with and without acute kidney injury. Intensive Care Med 36 1333-1340, 2010.

55. Paragas N, Qiu A, Zhang Q, et al: The Ngal reporter mouse detects the response of the kidney to injury in real time. Nat Med 17: 216-222, 2011.

56. Demirbilek S, Sizanli E, Karadag N, et al: The effects of methylene blue on lung injury in septic rats. Eur Surg Res 38: 35-41, 2006.

57. Ozdulger A, Cinel I, Koksel O, et al: The protective effect of $\mathrm{N}$-acetylcysteine on apoptotic lung injury in cecal ligation and puncture-induced sepsis model. Shock 19: 366-372, 2003.

58. Ma H, Kou J, Zhu D, Yan Y and Yu B: Liu-Shen-Wan, a traditional Chinese medicine, improves survival in sepsis induced by cecal ligation and puncture via reducing TNF-alpha levels, MDA content and enhancing macrophage phagocytosis. Int Immunopharmacol 6: 1355-1362, 2006.

59. Kahraman S, Kilinç K, Dal D and Erdem K: Propofol attenuates formation of lipid peroxides in tourniquet-induced ischaemia-reperfusion injury. Br J Anaesth 78: 279-281, 1997.

60. Soncul H, Oz E and Kalaycioglu S: Role of ischemic preconditioning on ischemia-reperfusion injury of the lung. Chest 115 1672-1677, 1999.

61. Xu B, Gao X, Xu J, et al: Ischemic postconditioning attenuates lung reperfusion injury and reduces systemic proinflammatory cytokine release via heme oxygenase 1 . J Surg Res 166: e157-e164, 2011.

62. Cinel I, Ark M, Dellinger P, et al: Involvement of Rho kinase (ROCK) in sepsis-induced acute lung injury. J Thorac Dis 4: 30-39, 2012

63. Kono Y, Inomata M, Hagiwara S, Shiraishi N, Noguchi T and Kitano S: A newly synthetic vitamin E derivative, E-Ant-S-GS, attenuates lung injury caused by cecal ligation and puncture-induced sepsis in rats. Surgery 151: 420-426, 2012.

64. Wu YX, Wu DW, Peng MM, Chen C, Lu HN and Zhao LK: The effect of low-dose hydrocortisone on the expression of glucocorticoid receptor alpha of the septic kidney and its protective effect on kidney in rat. Zhongguo Wei Zhong Bing Ji Jiu Yi Xue 23: 426-429, 2011 (In Chinese).

65. Yan GT, Xue H, Lin J, Hao XH, Zhang $\mathrm{K}$ and Wang LH: Leptin protects sepsis-induced renal injury and research for its mechanism. Zhongguo Wei Zhong Bing Ji Jiu Yi Xue 18: 665-667, 2006 (In Chinese).
66. Lee JA, Choi JW, In JH, et al: Hepatic ischemic preconditioning provides protection against distant renal ischemia and reperfusion injury in mice. J Korean Med Sci 27: 547-552, 2012.

67. Lee HT, Gallos G, Nasr SH and Emala CW: A1 adenosine receptor activation inhibits inflammation, necrosis, and apoptosis after renal ischemia-reperfusion injury in mice. J Am Soc Nephrol 15: 102-111, 2004.

68. van Lanschot JJ, Mealy K, Jacobs DO, Evans DA and Wilmore DW: Splenectomy attenuates the inappropriate diuresis associated with tumor necrosis factor administration. Surg Gynecol Obstet 172: 293-297, 1991.

69. Cunningham PN, Dyanov HM, Park P, Wang J, Newell KA and Quigg RJ: Acute renal failure in endotoxemia is caused by TNF acting directly on TNF receptor-1 in kidney. J Immunol 168: 5817-5823, 2002

70. Percival TJ and Rasmussen TE: Reperfusion strategies in the management of extremity vascular injury with ischaemia. Br J Surg 99 (Suppl 1): S66-S74, 2012.

71. Takeshita M, Tani T, Harada S, et al: Role of transcription factors in small intestinal ischemia-reperfusion injury and tolerance induced by ischemic preconditioning. Transplant Proc 42: 3406-3413, 2010

72. Oberholzer C, Oberholzer A, Clare-Salzler M and Moldawer LL: Apoptosis in sepsis: a new target for therapeutic exploration. FASEB J 15: 879-892, 2001.

73. Mainous MR, Ertel W, Chaudry IH and Deitch EA: The gut: a cytokine-generating organ in systemic inflammation? Shock 4: 193-199, 1995.

74. Holubec H, Payne CM, Bernstein H, et al: Assessment of apoptosis by immunohistochemical markers compared to cellular morphology in ex vivo-stressed colonic mucosa. J Histochem Cytochem 53: 229-235, 2005.

75. Gobe G: Identification of apoptosis in kidney tissue sections. Methods Mol Biol 466: 175-192, 2009.

76. Wu HH, Hsiao TY, Chien CT and Lai MK: Ischemic conditioning by short periods of reperfusion attenuates renal ischemia/reperfusion induced apoptosis and autophagy in the rat. J Biomed Sci 16: 19, 2009.

77. Perl M, Chung CS, Perl U, et al: Fas-induced pulmonary apoptosis and inflammation during indirect acute lung injury. Am J Respir Crit Care Med 176: 591-601, 2007.

78. Oliver L and Vallette FM: The role of caspases in cell death and differentiation. Drug Resist Updat 8: 163-170, 2005.

79. Jo SK, Cha DR, Cho WY, et al: Inflammatory cytokines and lipopolysaccharide induce Fas-mediated apoptosis in renal tubular cells. Nephron 91: 406-415, 2002.

80. Messmer UK, Briner VA and Pfeilschifter J: Tumor necrosis factor-alpha and lipopolysaccharide induce apoptotic cell death in bovine glomerular endothelial cells. Kidney Int 55: 2322-2337, 1999.

81. Du C, Guan Q, Yin Z, Zhong R and Jevnikar AM: IL-2-mediated apoptosis of kidney tubular epithelial cells is regulated by the caspase-8 inhibitor c-FLIP. Kidney Int 67: 1397-1409, 2005.

82. Hengartner MO: The biochemistry of apoptosis. Nature 407: 770-776, 2000.

83. Jun N, Ke J, Gang C, Lin C, Jinsong L and Jianjun W: The protective effect of ischemic preconditioning associated with altered gene expression profiles in rat lung after reperfusion. J Surg Res 168: 281-293, 2011. 